\title{
Lowest excited singlet states of $\alpha$-9-anthrylpolyenes
}

\author{
N. Holl, P. Emele, H. Port, H.C. Wolf \\ 3. Physikalisches Institut, Universität Stuttgart, Stuttgart, Germany \\ H. Strobel, T. Kesmarszky and F. Effenberger \\ Institut für Organische Chemie, Universität Stuttgart, Stuttgart, Germany
}

Received 16 November 1992; in final form 28 January 1993

\begin{abstract}
Properties of the electronically excited states of $\alpha$-9-anthrylpolyenes are studied in n-hexane solutions by using optical absorption, emission and fluorescence excitation spectroscopy. The absorption spectra reveal a complex structure with contributions of both anthracene and polyene type bands but also of bands with mixed anthracene/polyene character. Small amounts of remaining impurities are chemically not separable but can be detected via fluorescence measurements. By computer-aided decomposition of spectra measured under variable selective excitation the actual, clearly chain-length dependent, fluorescences could be obtained. The fluorescence quantum yield drastically decreases from $\phi_{\mathrm{f}}=0.75$ at $n=1$ to $\phi_{\mathrm{f}}=10^{-4}$ at $n=5$, whereas the radiative lifetime increases.
\end{abstract}

\section{Introduction}

The electronic properties of linear polyenes have been investigated extensively [1]. Current interests are directed particularly towards possible applications in model systems for both biophysics and future molecular electronic devices [2].

Symmetrically phenyl-substituted polyenes have been most widely used in previous studies because of higher stability and increased fluorescence quantum yields as compared to unsubstituted polyenes [3]. Their electronic properties reveal corresponding chain length dependencies related, however, to an extension of the electronic $\pi$ states into the substituents. Similar behaviour is found for systems based on symmetrical substitution with higher aromates, dinaphthylpolyenes [4] and dianthrylpolyenes $[5,6]$, and for systems like monophenylpolyenes [7]. Commonly singlet state properties of these compounds are investigated under conditions of isolated molecules in liquid or solid solution. Recently, we have contributed to this field by measurements of both singlet and triplet excited states for diphenylpolyenes in single crystal environment [8].
Within the context of the two active branches of research mentioned above asymmetrically substituted donor/acceptor-type polyenes are more relevant. Using anthracene as donor and various molecules as acceptor we have studied such systems with respect to the occurrence of intramolecular energy transfer [9-11] and charge separation [12].

As it turned out during that work, however, detailed understanding of the anthrylpolyenes themselves without acceptor substituent is required. These are appropriate as reference systems for the complex behaviour of the bi-substituted supermolecules. Therefore optical spectra of anthrylpolyenes $9 \mathrm{~A}-\mathrm{P}_{n}-$ $\mathrm{CH}_{3}$ are investigated for chain lengths $n \leqslant 5$. The major aim of the present work is to characterize the singlet state properties of these compounds and to identify the true fluorescence emissions for different chain lengths. Time-resolved measurements are presented elsewhere [12].

\section{Experimental}

The subject of our investigations were linear alltrans polyenes of the form $9 \mathrm{~A}-\mathrm{P}_{n}-\mathrm{CH}_{3}(n=1-5)$, 
see fig. 1a. The nomenclature used in this work is the

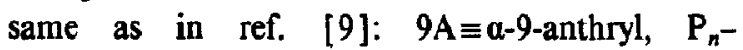
$\mathrm{CH}_{3} \equiv$ polyene chain of $n$ double bonds, which is terminally substituted by a methyl endgroup. Lateral methyl groups were introduced to stabilize the linear polyene chain. The compounds $9 \mathrm{~A}-\mathrm{P}_{n}-\mathrm{CH}_{3}$ were synthesized and extensively purified as described in refs. [13,14]. Remaining isomers (determined via HPLC) were below $0.5 \%$ for $n \geqslant 2.9 \mathrm{~A}-\mathrm{P}_{1}-\mathrm{CH}_{3}$ was a mixture of $83 \%$ trans-isomer and $17 \%$ cis-isomer. The samples were prepared as liquid solutions with a concentration of $10^{-6}-10^{-5} \mathrm{~mol} / \ell$ in order to avoid concentration effects and to maintain low optical densities. $n$-hexane (Uvasol, Merck) was used as purchased. The solutions were degassed from oxygen by repeated freeze-pump-thaw cycles. Optical absorption spectra were recorded at $T=295 \mathrm{~K}$ using a Perkin Elmer Lambda 16 UV/VIS spectrometer. Emission and excitation spectra were obtained at $T=180 \mathrm{~K}$ with a $450 \mathrm{~W}$ xenon lamp and a $0.25 \mathrm{~m}$ double monochromator as light source, and a $1 \mathrm{~m}$ double monochromator with cooled photomultiplier as detector. The photomultiplier was connected to a photon counter. All emission spectra in this work are corrected for the spectral responses of the experimental equipment and represent quantum fluxes. Quantum yields were measured relative to a common standard, anthracene in $n$-hexane $\left(\phi_{\mathrm{f}}=0.30\right.$ at $T=295 \mathrm{~K}[15], \phi_{\mathrm{f}}=0.55$ at $\left.T=180 \mathrm{~K}\right)$.

\section{Results and discussion}

The absorption spectra of the anthrylpolyenes $(n=1-5)$ are shown in fig. 1b. They consist of several bands, which vary in absorption strength and partly are displaced as a function of chain length. The main absorption band is found in all spectra at about $39000 \mathrm{~cm}^{-1}$, which is characteristic for the anthracene $S_{0} \rightarrow S_{3}$ transition. With increasing chain length, however, the extinction coefficient of this band is reduced (from $n=1$ to $n=5$ by about a factor of 2 ).

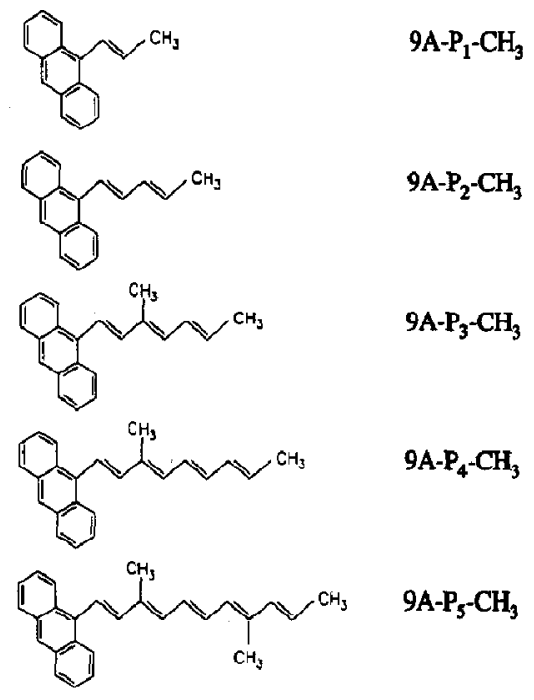

(a)

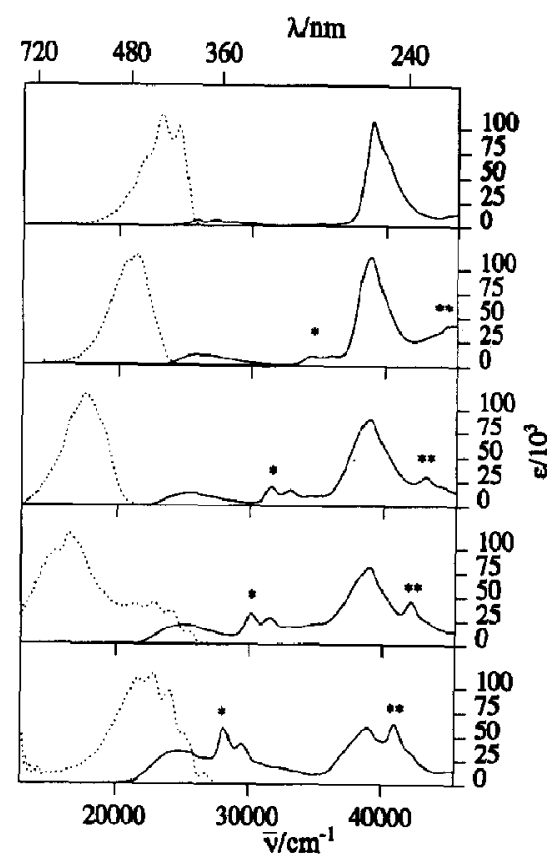

(b)

Fig. 1. (a) Chemical structure of the compounds $9 \mathrm{~A}-\mathrm{P}_{n}-\mathrm{CH}_{3}$ investigated. (b) (-) Absorption spectra. $T=295 \mathrm{~K}$. and - indicate new absorption bands (see text). ( $\cdots$ ) Emission spectra, $T=180 \mathrm{~K}$, normalized to the same maximum intensity. Excitation wavelength was $257 \mathrm{~nm}$. 
Instead the low energy $S_{0} \rightarrow S_{1}$ absorption band intensifies with chain length and is red-shifted from about 29000 to $24000 \mathrm{~cm}^{-1}$. For $n \geqslant 2$ additional absorption bands (labelled * and **, respectively, in fig. lb) appear in the spectra. They also intensify with $n$ and are red-shifted, but cannot be attributed to the anthracene or polyene moiety. As a detailed theoretical analysis revealed [16], these new bands correspond to mixed electronic transitions involving both anthracene and polyene states changing the predominant character with increasing $n$.

The fluorescence emission spectra of the anthrylpolyenes after excitation in the main absorption band at $39000 \mathrm{~cm}^{-1}$ are also given in fig. $1 \mathrm{~b}$. These spectra are strongly red-shifted with increasing $n$ and are corresponding for $n \leqslant 3$ in energetic position to the lowest absorption band. For $n=4$ the spectrum seems to consist of at least two contributions, the one at lower energy with its maximum position fitting well in the sequence $n=1-3$ but the other one overlapping with the absorption. For $n=5$ even the main portion of the fluorescence is located in the blue spectral region and overlapping with the absorption.

Upon varying the excitation energy it is found that the emission spectra for $n \leqslant 3$ consist of one single component as shown in fig. $1 \mathrm{~b}$, whereas those for $n=4$ and $n=5$ strongly depend on the excitation. As an example the fluorescence spectra for $n=4$ obtained at different excitation wavelengths are plotted in fig. 2. Obviously the fluorescence emission exhibits several spectral components whose relative contributions to the overall spectra vary with the ex-

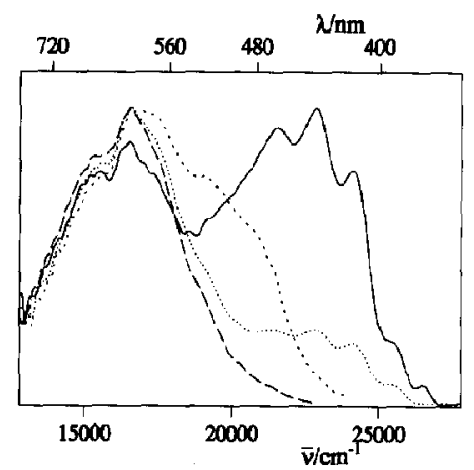

Fig. 2. Fluorescence spectra of $9 \mathrm{~A}-\mathrm{P}_{4}-\mathrm{CH}_{3}$ at different excitation wavelengths $(320 \mathrm{~nm}(\cdots), 340 \mathrm{~nm}(-), 410 \mathrm{~nm}$ $(--)$ and $430 \mathrm{~nm}(---))$. The spectra are normalized to the same maximum intensity. citation. Correspondingly, different fluorescence excitation spectra are observed for different detection wavelengths. These observations are supported by time-resolved spectroscopy [12]. The fluorescences reveal monoexponential decays for $n \leqslant 3$, but bi- or multi-exponential decays for $n=4$ and $n=5$.

In order to identify the actual fluorescence components of the $n=4$ and $n=5$ species and to separate quantitatively the different spectral contributions a spectral decomposition has to be performed. We applied a principal component analysis (PCA) ${ }^{\# 1}$ to the observed spectra.

Two examples for the decomposition of the fluorescence spectra at $n=4$ (with $\lambda_{\text {ex }}=340$ and $430 \mathrm{~nm}$, respectively, see also fig. 2) are provided in fig. 3. The analysis leads to four principal components, whose relative contributions were obtained by fitting their weighted sum to the observed spectra. In the upper parts of figs. $3 a$ and $3 b$ the fit is demonstrated.

There are no means to identify directly the four principal components obtained. However, qualita-

-1 From the various emission spectra of one sample a correlation matrix is built and diagonalized. Then a varimax rotation [17] is performed on the main eigenvectors. The resulting vectors can be interpreted as spectral components which are superimposed to form the original emission spectra.

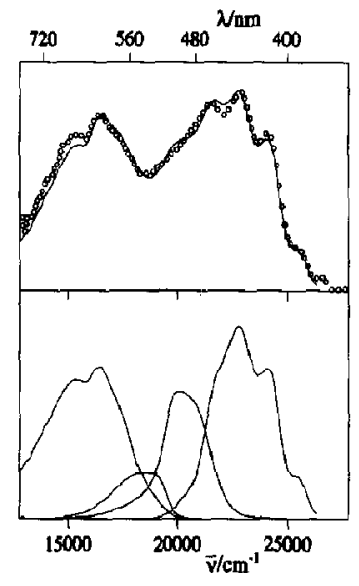

(a)

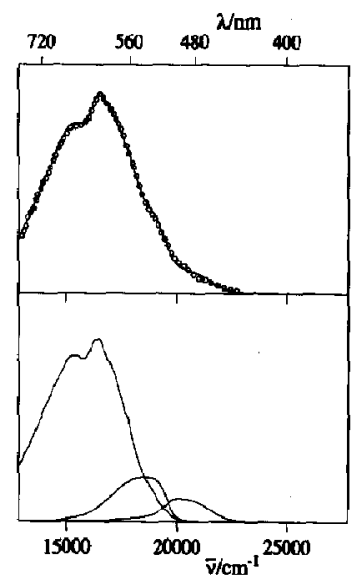

(b)
Fig. 3. Two examples ( (a) and (b) ) for the decomposition of the $9 \mathrm{~A}-\mathrm{P}_{4}-\mathrm{CH}_{3}$ emission, recorded at an excitation wavelength of 340 and $430 \mathrm{~nm}$, respectively (spectra 2 and 4 in fig. 2). Top: emission spectrum $(O)$ and superposition of principal compo-s nents (-). Bottom: weighted principal components. 
tively they can be categorized as follows. The fourth component at lowest energy is assumed to represent the actual $n=4$ fluorescence in good approximation, since it is obtained after long-wavelength excitation and apparently, compared with figs. 1 and 3, could fit with respect to its spectral characteristics within the dependence on $n$ stated above for $n \leqslant 3$. For the other three principal components a striking resemblance to the fluorescence spectra of shorter polyenes for $n \leqslant 3$ is discovered, when normalizing the spectra taken from figs. 1 and 3 to the same intensity (fig. 4). From the close similarity of the spectral features we are led to the conclusion that impurities of similar molecular composition must be involved and are causing the additional emissions. Further arguments about the possible nature of these impurities will be discussed below.

For the case $n=5$ an extended purification [13] led to a strongly reduced overall fluorescence quantum yield, confirming the presence of impurities. The

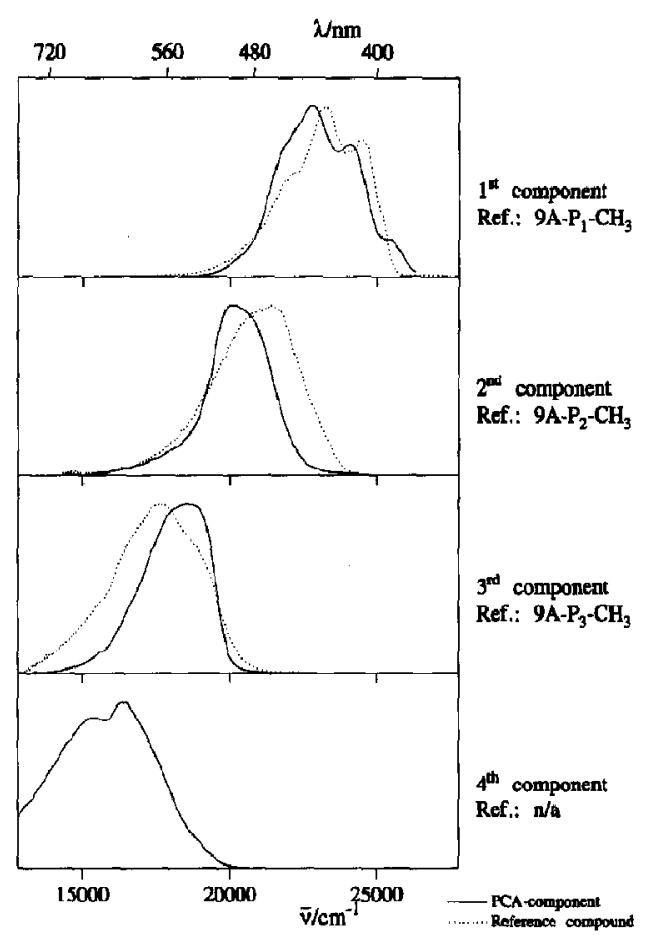

Fig. 4. The principal components of the $9 \mathrm{~A}-\mathrm{P}_{4}-\mathrm{CH}_{3}$ emission (-) are shown together with the fluorescence of reference compounds ( . - ) . From top to bottom; 1 st component / reference $9 \mathrm{~A}-\mathrm{P}_{1}-\mathrm{CH}_{3}$, 2nd component / $9 \mathrm{~A}-\mathrm{P}_{2}-\mathrm{CH}_{3}$, 3rd component $/ 9 \mathrm{~A}-\mathrm{P}_{3}-\mathrm{CH}_{3}$, 4 th component $/$ no reference.
PCA method applied to the fluorescences yielded five components. Out of these, the one at lowest energy is assigned to the actual fluorescence of $9 \mathrm{~A}-\mathrm{P}_{5}-\mathrm{CH}_{3}$, whereas the other components like in the case of $n=4$ are similar to the spectral components of the shorter anthrylpolyenes.

After separation of the actual fluorescence contributions of the species $n=4$ and $n=5$ it was possible to determine the fluorescence quantum yield $\phi_{\mathrm{f}}$ for the whole series of $9 \mathrm{~A}-\mathrm{P}_{n}-\mathrm{CH}_{3}$, with $n=1-5$. The values of $\phi_{\mathrm{f}}$ are plotted in fig. 5 as a function of $n$. They drastically decrease with increasing chain length (from $\phi_{\mathrm{r}}=0.75$ at $n=1$ to $\phi_{\mathrm{f}}=10^{-4}$ at $n=5$ ). By using the measured fluorescence lifetimes [12] the radiative lifetimes have been determined and the values $\tau_{\text {rad }}(n)$ are plotted in fig. 5. Formally, as an additional reference compound for $n=0\left(9 \mathrm{~A}-\mathrm{P}_{0^{-}}\right.$ $\mathrm{CH}_{3}$ ), the experimental values $\phi_{f}$ and $\tau_{\text {rad }}$ for 9 methylanthracene have been included in the plots of fig. 5. For $n=1,2$ there are no significant changes in $\phi_{\mathrm{f}}$ and $\tau_{\text {rad }}$ compared to $n=0$. This could be explained by the lowest excited singlet state having strong anthracene-like properties.

It must be emphasized that the essential changes in $\phi_{\mathrm{f}}$ and $\tau_{\mathrm{rad}}$ occur at $n>2$ (corresponding to the results on monophenylpolyenes [7]) when an increasing energy gap appears between absorption and fluorescence. These aspects are treated theoretically in ref. [16]. The observed radiative lifetime $\tau_{\text {rad }}$ increases with $n$, whereas the one obtained from the oscillator strength of the lowest absorption band decreases. A similar discrepancy is reported in the literature [1] for various polyene systems, where it is attributed to the fact that the fluorescing singlet state

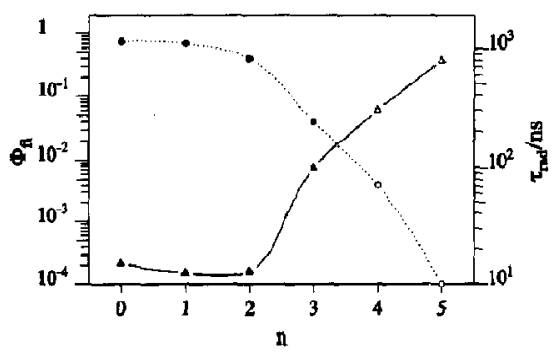

Fig. 5. Fluorescence quantum yields $\phi_{f}(0,0)$ and radiative decay times $\tau_{\text {rad }}(\Delta, \Delta)$ of the compounds $9 \mathrm{~A}-\mathrm{P}_{n}-\mathrm{CH}_{3}(n=0-5)$ in $n$-hexane at $T=180 \mathrm{~K}$. Non-shaded symbols indicate values obtained for the characteristic component of the PCA result (see text). 
of polyenes with $n>3$ is of the same symmetry as the ground state and therefore forbidden in absorption. The same explanation seems to be applicable to the present case. It is consistent with the observed energy gap between absorption and fluorescence and with the increase in $\tau_{\text {rad. }}$ The observed behaviour for $n>2$ could be explained by the low-lying excited singlet states having strong polyene-like properties.

In summary, the overall chain length dependence of the observed energy gap and of $\tau_{\text {rad }}$ for $9 \mathrm{~A}-\mathrm{P}_{n}-\mathrm{CH}_{3}$ ( $n=1-5$ ) can be explained by assuming the lowest excited singlet state to change from anthracene-like $(n \leqslant 2)$ to polyene-like $(n>2)$.

As emphasized above, the impurities causing the high-energy components in the fluorescence spectra could not be definitely identified. In the following, however, further arguments about their possible nature will be summarized.

(i) Additionally, fluorescence excitation spectra of the individual impurity emissions have been studied. They were obtained indirectly in two steps since the impurity emissions were spectrally overlapping. First, series of fluorescence spectra were measured under variable, energy selective, excitation (in the range between 20000 and $35000 \mathrm{~cm}^{-1}$ ) and constant excitation intensity. Second, these spectra were decomposed applying the PCA as above. Combining these two sets of data, fluorescence excitation spectra were derived, point by point, which correspond to the absorption spectra of the anthrylpolyenes and clearly exhibit the same chain length dependency. Thus, these spectra reveal a close similarity between the impurities and short chain anthrylpolyenes as stated above. Isomers could be excluded, because the resulting fluorescence excitation spectra were clearly different from absorption spectra to be expected for isomers (these should be similar to the absorption spectra of the original compounds except for slight shifts).

(ii) When attributing the fluorescence components obtained by the PCA to individual impurity molecules, the impurity concentrations can be estimated. The values obtained from the relative quantum yields in the fluorescence spectra are in the range $1 \%-3 \%$.

(iii) Isomerization during the optical experiment as possible source for impurities was ruled out by comparing HPLC before and after the measure- ments. Photochemistry during the measurements was not observed, there was quantitatively no difference between the spectra obtained at the beginning and at the end of the experiment.

Photochemically produced impurities are found only under extreme conditions upon irradiating the sample in the presence of oxygen (which otherwise is excluded during the experiment) for several hours at light intensities increased by orders of magnitude.

\section{Summary}

In this Letter, absorption and fluorescence spectra of anthrylpolyenes $9 \mathrm{~A}-\mathrm{P}_{n}-\mathrm{CH}_{3}$ have been studied as a function of chain length $(n=1-5)$. The measurements of the emission spectra required particular effort to separate the true fluorescences of the compounds from those of residual impurities (presumably compounds similar to anthrylpolyenes with shorter chain lengths carrying relatively high quantum yields).

The fluorescence quantum yield of $9 \mathrm{~A}-\mathrm{P}_{n}-\mathrm{CH}_{3}$ diminishes for an increasing number $n$ of double bonds, whereas the observed radiative lifetime increases. At the same time an energy gap between absorption and fluorescence appears. This behaviour can be explained assuming the lowest excited singlet state changing its properties from anthracene-like $(n \leqslant 2)$ to polyene-like $(n>2)$.

\section{Acknowledgement}

We gratefully acknowledge financial support from the Deutsche Forschungsgemeinschaft (SFB 329) and from the Fonds der Chemischen Industrie.

\section{References}

[1] B.S. Hudson, B.E. Kohler and K. Schulten, in: Excited states, Vol. 6, ed. E.C. Lim (Academic Press, New York, 1982).

[2] F.L. Carter, ed., Molecular electronic devices I/II (Dekker, New York 1982/1987).

[3] B.E. Kohler, in: Conjugated polymers: the novel science and technology of conducting and nonlinear optically active materials, eds. J.L. Bredas and R. Silbey (Kluwer, Dordrecht, 1991 ) p. 405. 
[4] A. Yasuhara, S. Akiyama and M. Nakagawa, Bull. Chem. Soc. Japan 45 (1972) 3638.

[5] Y. Takeuchi, S. Akiyama and M. Nakagawa, Bull. Chem. Soc. Japan 45 (1972) 3183.

[6] K.G. Mandal and T.N. Misra, Bull. Chem. Soc. Japan 49 (1976) 198.

[7] W.J. Buma, B.E. Kohler, J.M. Nuss, T.A. Shaler and K. Song, J. Chem. Phys. 96 (1992) 4860.

[8] V. Weiss, H. Port and H.C. Wolf, Chem. Phys. Letters 192 (1992) 289.

[9] G. Blessing, N. Holl, H. Port, H.C. Wolf, F. Effenberger, Th. Kesmarszky and H. Schlosser, Mol. Cryst. Liquid Cryst. $183(1990) 21$.
[10] F. Effenberger, H. Schlosser, P. Bäuerle, St. Maier, H. Port and H.C. Wolf, Angew. Chem. 100 (1989) 274.

[11] St. Maier, H. Port, H.C. Wolf, F. Effenberger and $H$. Schlosser, Synth. Metals 29 (1989) 517(E).

[12] G. Quapil, Dissertation, Universităt Stuttgart (1992).

[13] H. Strobel, Dissertation, Universität Stuttgart (1992).

[14] T. Kesmarszky, Dissertation, Universität Stuttgart (1990).

[15] S. Ates and A. Yildiz, J. Chem. Soc. Faraday Trans. I 79 (1983) 2853.

[16] P. Gribi, Dissertation, Universität Stuttgart (1992).

[17] IBM System/360 Scientific Subroutine Package, Version III, Programmer's Manual (1968). 